Gut and Liver, Vol. 10, No. 4, July 2016, pp. 532-541

\title{
Magnifying Endoscopy with Narrow Band Imaging of Early Gastric Cancer: Correlation with Histopathology and Mucin Phenotype
}

Kyung-Sun Ok ${ }^{1}$, Gwang Ha Kim², Do Youn Park ${ }^{3}$, Hyun Jeong Lee ${ }^{2}$, Hye Kyung Jeon², Dong Hoon Baek ${ }^{2}$, Bong Eun Lee ${ }^{2}$, and Geun Am Song ${ }^{2}$

${ }^{1}$ Department of Internal Medicine, Inje University Busan Paik Hospital, Inje University College of Medicine, Departments of ${ }^{2}$ Internal Medicine and ${ }^{3}$ Pathology, Pusan National University School of Medicine and Biomedical Research Institute, Pusan National University Hospital, Busan, Korea

Background/Aims: Magnifying endoscopy with narrow band imaging (ME-NBI) is a useful modality for the detailed visualization of microsurface (MS) and microvascular (MV) structures in the gastrointestinal tract. This study aimed to determine whether the MS and MV patterns in ME-NBI differ according to the histologic type, invasion depth, and mucin phenotype of early gastric cancers (EGCs). Methods: The MS and MV patterns of 160 lesions in 160 patients with EGC who underwent ME-NBI before endoscopic or surgical resection were prospectively collected and analyzed. EGCs were categorized as either differentiated or undifferentiated and as either mucosal or submucosal, and their mucin phenotypes were determined via immunohistochemistry of the tumor specimens. Results: Differentiated tumors mainly displayed an oval and/or tubular MS pattern and a fine network or loop MV pattern, whereas undifferentiated tumors mainly displayed an absent MS pattern and a corkscrew MV pattern. The destructive MS pattern was associated with submucosal invasion, and this association was more prominent in the differentiated tumors than in the undifferentiated tumors. MUC5AC expression was increased in lesions with either a papillary or absent MS pattern and a corkscrew MV pattern, whereas MUC6 expression was increased in lesions with a papillary MS pattern and a loop MV pattern. CD10 expression was more frequent in lesions with a fine network MV pattern. Conclusions: ME-NBI can be useful for predicting the histopathology and mucin phenotype of EGCs. (Gut Liver 2016;10:532-541)

Key Words: Magnifying endoscopy; Narrow band imaging; Stomach neoplasms; Mucins

\section{INTRODUCTION}

Magnifying endoscopy (ME) with narrow band imaging (MENBI) is a useful modality for detailed visualization of microstructures and microvessels within the superficial layer of the gastric mucosa. Yao et al. ${ }^{1}$ first reported unique ME findings based mainly on the subepithelial microvascular (MV) architecture characteristics of differentiated-type early gastric cancers (EGCs). ME-NBI was subsequently reported to be capable of predicting the histological characteristics of EGCs; a fine network or intralobular pattern suggests differentiated-type carcinomas and a corkscrew pattern suggests undifferentiated-type carcinomas. ${ }^{2-4}$ However, these studies had limitations; many lesions had unclassified patterns and the patterns were mainly based on MV structure. Although ME-NBI is useful for predicting the invasion depth of esophageal and colon cancers, ${ }^{5-8}$ its usefulness for determining the depth of gastric cancer have not been clearly elucidated. ${ }^{9,10}$

Recent advances in mucin immunohistochemistry have facilitated the goal of classifying gastric neoplasms according to mucin phenotype. Among the various mucin markers, MUC5AC, MUC2, MUC6, and CD10 are generally used to classify gastric cancers. Several studies have suggested that the type of mucin expressed in early and advanced gastric carcinomas is of clinical significance, alluding to biologic differences in precursor lesions and/or pathways of malignant transformation. ${ }^{11-14}$ In our previous study, the mucin phenotype was significantly correlated with size, depth of invasion, macroscopic type, and histologic type of tumor. ${ }^{12}$ Patients with EGC whose tumors had a gastric mucin phenotype survived longer compared to those with an intestinal mucin phenotype. However, despite many investiga-

Correspondence to: Gwang Ha Kim

Department of Internal Medicine, Pusan National University School of Medicine and Biomedical Research Institute, Pusan National University Hospital, 179 Gudeok-ro, Seo-gu, Busan 49241, Korea

Tel: +82-51-240-7869, Fax: +82-51-244-8180, E-mail: doc0224@pusan.ac.kr

Received on August 2, 2015. Revised on September 11, 2015. Accepted on October 6, 2015. Published online March 30, 2016

pISSN 1976-2283 eISSN 2005-1212 http://dx.doi.org/10.5009/gnl15364

@ This is an Open Access article distributed under the terms of the Creative Commons Attribution Non-Commercial License (http://creativecommons.org/licenses/by-nc/4.0) which permits unrestricted non-commercial use, distribution, and reproduction in any medium, provided the original work is properly cited. 
tions, the clinical validity of the mucin phenotype in gastric cancer remains controversial. ${ }^{12,15-18}$ Different microsurface (MS) and MV patterns are apparent in ME-NBI of EGCs, and it is possible that different patterns denote different mucin phenotype. However, to date, few studies have addressed this possibility. ${ }^{19}$ Therefore, the present study aimed to determine whether the MS and MV patterns in ME-NBI differ according to the histologic type, invasion depth, and mucin phenotype of EGCs.

\section{MATERIALS AND METHODS}

\section{Patients}

From June 2012 to May 2014, a total of 185 patients with an endoscopic diagnosis of EGC underwent ME-NBI. Of these patients, 25 were excluded from our study for the following reasons: the absence of final histological results because of follow-up loss ( $n=3)$, advanced gastric cancer after surgery $(n=7)$, or subpar image quality owing to the patient's noncooperation or severe ulceration with thick exudate $(n=15)$. As a result, a total of 160 EGCs from 160 patients (113 men and 47 women; age range, 31 to 84 years; median age, 61 years) were included in this study. ME-NBI data were prospectively collected and analyzed, with special attention to the MS and MV patterns of each lesion. The study protocol was reviewed and approved by the Institutional Review Board of the Pusan National University Hospital (E-2014136).

\section{ME-NBI}

The Evis Lucera Spectrum system (Olympus Co., Tokyo, Japan), comprising a light source (CLV-260SL), a processor (CV260SL), and a magnifying video endoscope (GIF-H260Z), was used as the video endoscopy system. This system was equipped with both white light and NBI modes, which could be toggled using a button on the control head of the video endoscope. This system can reach zoom magnifications of $\times 80$. To obtain a clear view for ME-NBI, a soft hood (MB-46; Olympus Co.) was fitted on the distal tip of the endoscope to maintain the focal distance.

ME-NBI was performed by a single experienced endoscopist (G.H.K.) who had previously performed over 100 ME-NBI examinations. All examinations were performed under conscious sedation with 2 to $5 \mathrm{mg}$ of midazolam. After routine observation, ME-NBI examinations of EGC areas were performed to evaluate the MS and MV patterns. MS patterns were classified as oval and/or tubular, papillary, destructive, or absent, while MV patterns were classified as loop, fine network, or corkscrew (Fig. 1). ${ }^{2,4,20}$ If lesions showed combined MS or MV patterns, the predominant pattern was used for classification.

\section{Histological assessment}

Endoscopic submucosal dissection (ESD) or gastrectomy was performed within 2 weeks after ME-NBI. The resected specimens were fixed in 10\% buffered formalin. Carcinomas and adjacent nonneoplastic mucosa were serially cut into 2-mm slices in parallel, embedded in paraffin, sectioned, and stained with he-

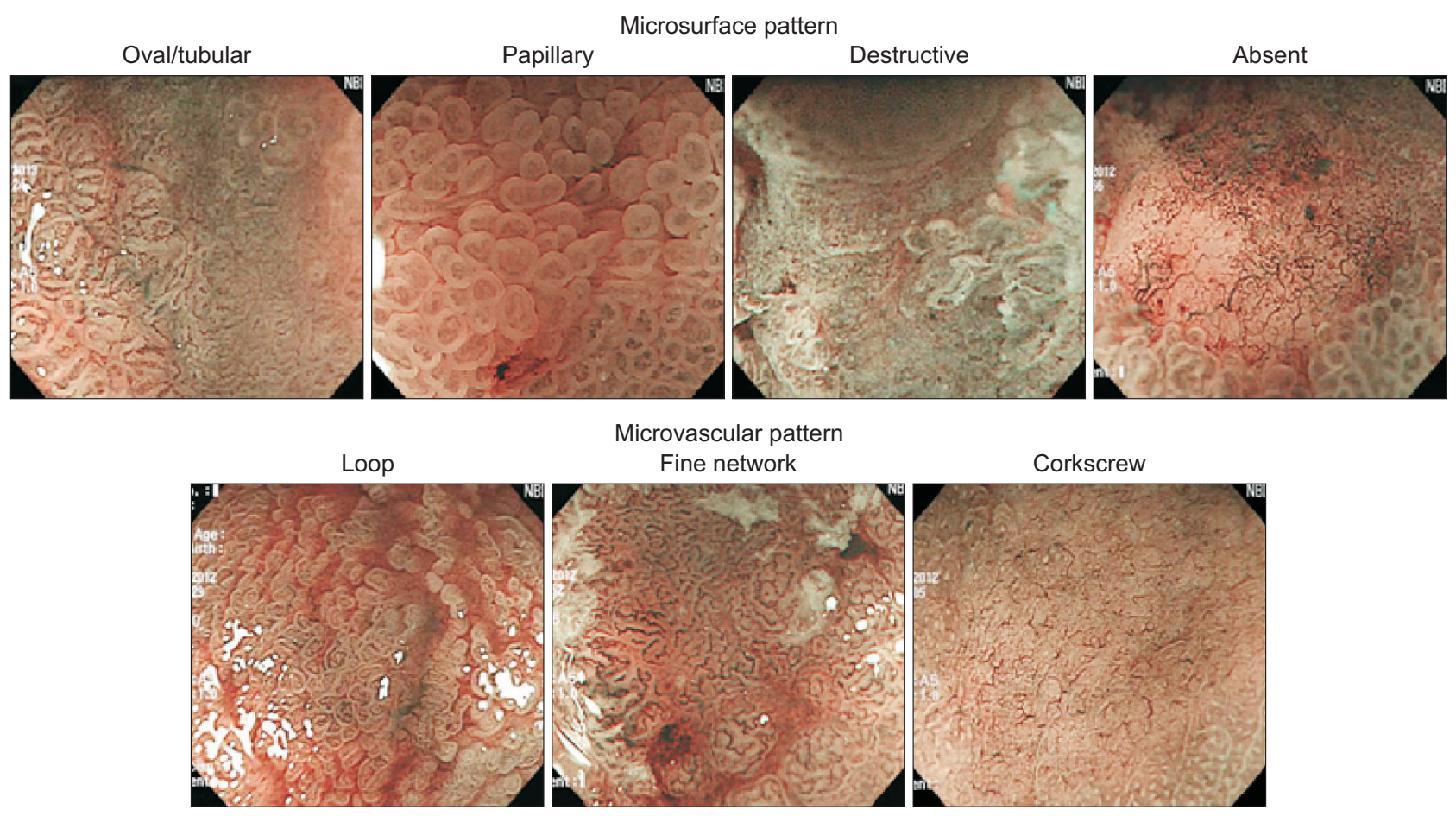

Fig. 1. Classification of microsurface and microvascular patterns using magnifying endoscopy with narrow band imaging in early gastric cancers. 
matoxylin and eosin for histological examination. Tumor size, depth of invasion, presence of ulceration, degree of differentiation, and lymphovascular invasion were evaluated microscopically according to the Japanese Classification of Gastric Carcinomas. $^{21}$

\section{Mucin phenotype}

Immunohistochemical expression of MUC2 (Сcp58, 1:500; Novocastra Laboratories, Newcastle, UK), MUC5AC (CLH2, 1:500; Novocastra Laboratories), MUC6 (CLH5, 1:500; Novocastra Laboratories), and CD10 (56C6, 1:100; Novocastra Laboratories ) was evaluated in the cancer cells. ${ }^{22}$ Briefly, 5 - $\mu$ m-thick consecutive tumor sections were deparaffinized and hydrated through a graded series of alcohol concentrations. Antigen retrieval was performed in $10 \mathrm{mmol} / \mathrm{L}$ citrate buffer ( $\mathrm{pH} \mathrm{6.0)} \mathrm{in} \mathrm{a} \mathrm{microwave}$ oven for 10 minutes, after which endogenous peroxidase activity was inhibited by immersion of the sections in a $3 \% \mathrm{H}_{2} \mathrm{O}_{2}$ / methanol solution. The sections were incubated with the primary antibodies followed by thorough washing in phosphatebuffered solution. Next, they were incubated successively with biotinylated secondary antibody and avidin-biotinylated horseradish peroxidase complex (Vectastain Elite ABC kit; Vector Laboratories, Burlingame, CA, USA). Antibody binding was visualized using 3,3'-diaminobenzidine tetrachloride as the chromogen, and nuclei were counterstained with Mayer's hematoxylin. Immunostaining was considered positive if $\geq 10 \%$ of the cancer cells were immunoreactive as previously reported. ${ }^{11,22}$

MUC5AC, MUC6, MUC2, and CD10 have been found to be specifically expressed in the gastric foveolar epithelium, pyloric gland cells, goblet cells, and brush border, respectively. ${ }^{12}$ Mucin phenotypes were divided into four types, namely, the gastric (Gtype), intestinal (I-type), gastrointestinal (GI-type), and null (Ntype), depending on the expression of gastric mucins (MUC5AC and MUC6) and intestinal mucins (MUC2 and CD10). Gastric cancers in which more than 10\% of the section area expressed at least one gastric or intestinal mucin marker were classified as the G-type or I-type, respectively. Gastric cancers that exhibited both gastric and intestinal mucin expression were classified as the GI-type, while those showing neither gastric nor intestinal mucin expression were classified as the N-type.

\section{Statistical analysis}

Differences in MS and MV patterns according to histologic type, invasion depth, or mucin phenotype were evaluated using the chi-square test or Fisher exact test. Statistical calculations were performed using the IBM SPSS version 21.0 for Windows (IBM Co., Armonk, NY, USA). Results were considered statistically significant when the $\mathrm{p}$-value was $<0.05$.

\section{RESULTS}

\section{Clinicopathologic characteristics of EGCs}

Of the 160 EGC lesions examined in this study, 23 were located in the upper third, 78 in the middle third, and 59 in the lower third of the stomach. Macroscopically, the most common lesion shape was the depressed shape (93/160, 58.1\%). Sixty-six lesions were treated via ESD, and 110 lesions were treated via surgery. The results of the final histologic assessment in regard to histologic type and invasion depth were as follows: 105 lesions were differentiated-type and 55 were undifferentiatedtype, and 110 lesions were mucosal cancers and 50 were submucosal cancers (Table 1). In regard to mucin phenotype, 85 lesions were G-type, 32 were GI-type, 28 were I-type, and 16 were N-type.

Table 1. Baseline Clinicopathologic Characteristics of Early Gastric Cancers

\begin{tabular}{|c|c|}
\hline Characteristic & Value \\
\hline No. of patients & 160 \\
\hline Age, yr & $61(31-84)$ \\
\hline \multicolumn{2}{|l|}{ Sex } \\
\hline Male & $113(70.6)$ \\
\hline Female & 47 (29.4) \\
\hline \multicolumn{2}{|l|}{ Location } \\
\hline Upper third & $23(14.4)$ \\
\hline Middle third & 78 (48.8) \\
\hline Lower third & 59 (36.9) \\
\hline \multicolumn{2}{|l|}{ Macroscopic shape } \\
\hline Elevated & 51 (31.9) \\
\hline Flat & $16(10.0)$ \\
\hline Depressed & $93(58.1)$ \\
\hline Size, mm & $26(6-90)$ \\
\hline \multicolumn{2}{|l|}{ Histologic type } \\
\hline Differentiated-type & $105(65.6)$ \\
\hline Undifferentiated-type & $55(34.4)$ \\
\hline \multicolumn{2}{|l|}{ Mucin phenotype } \\
\hline Gastric type & $85(53.1)$ \\
\hline Gastrointestinal type & $31(19.4)$ \\
\hline Intestinal type & $28(17.5)$ \\
\hline Null type & $16(10.0)$ \\
\hline \multicolumn{2}{|l|}{ Depth of invasion } \\
\hline Mucosa & $110(68.8)$ \\
\hline Submucosa & $50(31.3)$ \\
\hline \multicolumn{2}{|l|}{ Treatment } \\
\hline Endoscopic resection & $55(34.4)$ \\
\hline Surgery & $105(65.6)$ \\
\hline
\end{tabular}

Data are presented as number (\%) or mean (range). 

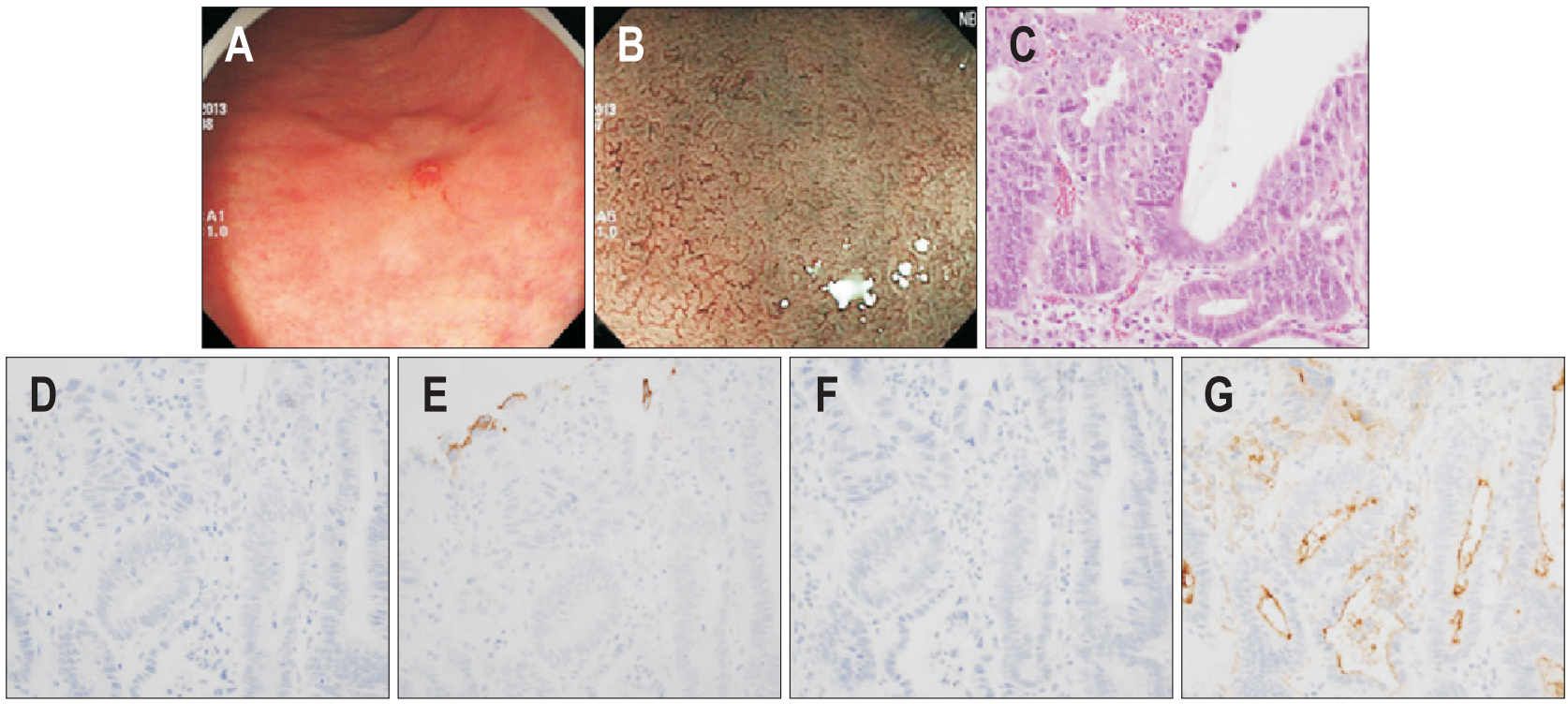

Fig. 2. A case of differentiated cancer. (A) A depressed lesion is observed in the lower body. (B) Magnifying endoscopy with narrow band imaging indicates an oval and/or tubular microsurface and fine network microvascular patterns. (C) Histologic examination shows a well-differentiated adenocarcinoma in the lamina propria (H\&E stain, $\times 40$ ). The cancer cells are strongly positive for CD10 (G) and negative for MUC2 (D), MUC5AC (E) and MUC6 (F).

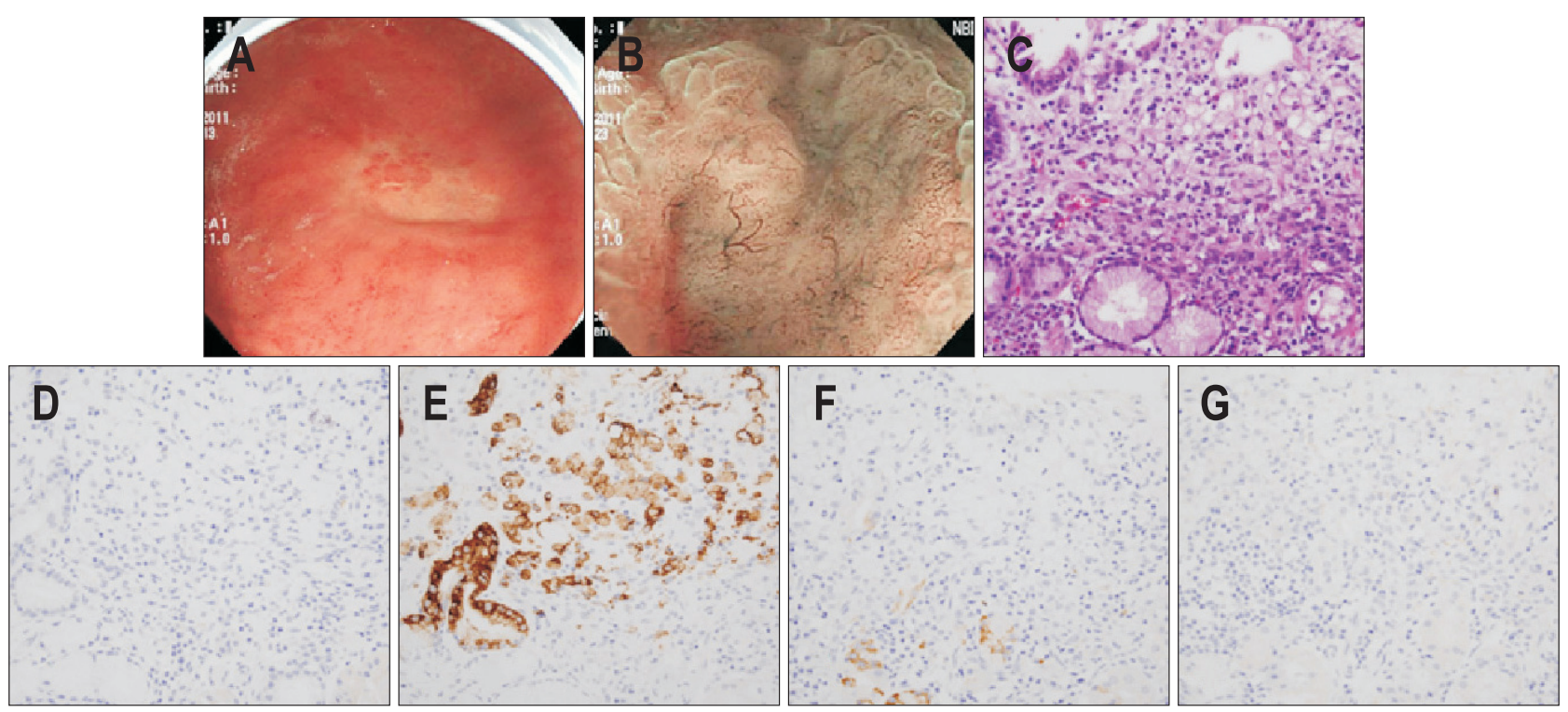

Fig. 3. A case of undifferentiated cancer. (A) A depressed lesion is observed in the lower body. (B) Magnifying endoscopy with narrow band imaging indicates an absence of microsurface and corkscrew microvascular patterns. (C) Histologic examination shows a signet ring cell carcinoma in the lamina propria (H\&E stain, $\times 40)$. The cancer cells are strongly positive for MUC5AC (E) and negative for MUC2 (D), MUC6 (F) and CD10 (G).

\section{MS and MV patterns of EGCs according to histologic type}

MS patterns were analyzed according to histologic type (Figs 2 and 3). In differentiated-type EGCs, an oval and/or tubular pattern was observed in 65 lesions (61.9\%), a destructive pattern in 22 lesions (21.0\%), a papillary pattern in 13 lesions (12.4\%), and an absent pattern in five lesions (4.8\%) (Table 2). In undif- ferentiated-type EGCs, an absent MV pattern was observed in 24 lesions (43.6\%), an oval and/or tubular pattern in 18 lesions (32.7\%), a destructive pattern in 12 lesions (21.8\%), and a papillary pattern in one lesion (1.8\%). Therefore, the main MS patterns were oval and/or tubular in differentiated-type EGCs and absent in undifferentiated-type EGCs ( $\mathrm{p}<0.001)$.

MV patterns were also analyzed according to histologic type. In differentiated-type EGCs, a fine network pattern was ob- 
Table 2. Magnifying Endoscopy with Narrow Band Imaging of Early Gastric Cancers according to the Histologic Type of the Tumor

\begin{tabular}{lccc}
\hline & Differentiated-type $(\mathrm{n}=105)$ & Undifferentiated-type $(\mathrm{n}=55)$ & $\mathrm{p}$-value \\
\hline Microsurface pattern & $65(61.9)$ & & $<0.001$ \\
Oval and/or tubular & $13(12.4)$ & $1(32.7)$ & $12(21.8)$ \\
Papillary & $22(21.0)$ & $24(43.6)$ & $<0.001$ \\
Destructive & $5(4.8)$ & $2(3.6)$ & $3(5.5)$ \\
Absent & & $50(90.9)$ & \\
Microvascular pattern & $43(41.0)$ & \\
Loop & $50(47.6)$ & \\
Fine network & $12(11.4)$ & \\
Corkscrew & &
\end{tabular}

Data are presented as number (\%).
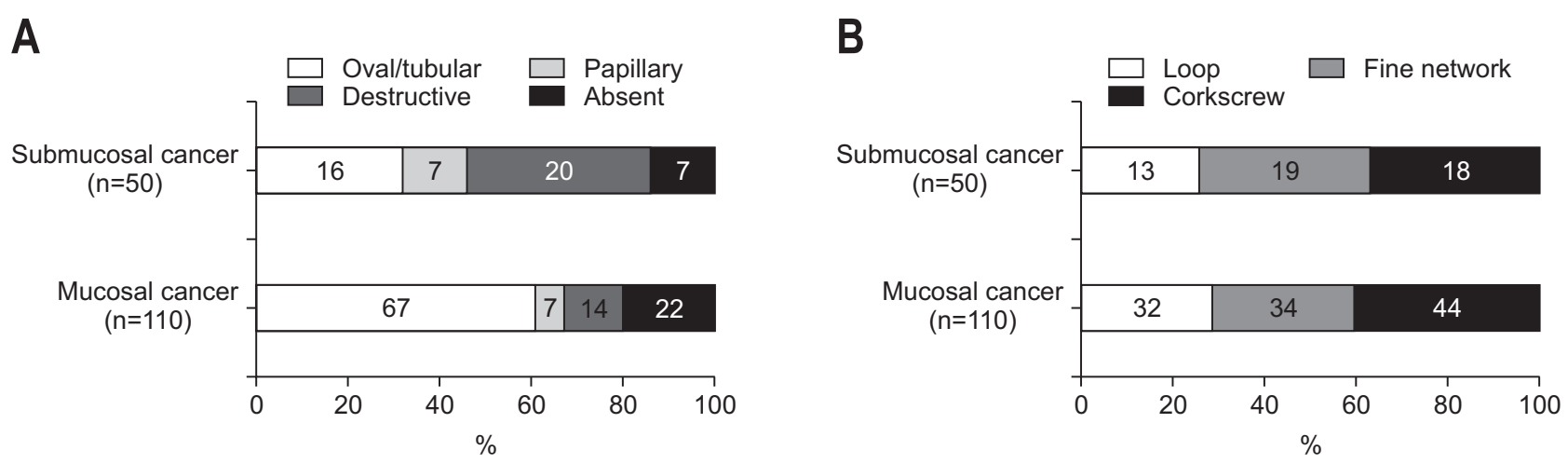

Fig. 4. Magnifying endoscopy with narrow band imaging of early gastric cancers according to the invasion depth of the tumor. (A) The main microsurface pattern in mucosal cancers was oval and/or tubular, and the main microsurface pattern in submucosal cancers was destructive ( $\mathrm{p}<0.001$ ). (B) There was no difference in the microvascular patterns between mucosal and submucosal cancers $(\mathrm{p}=0.677)$.

served in 50 lesions (47.6\%), a loop pattern in 43 lesions (41.0\%), and a corkscrew pattern in 12 lesions (11.4\%) (Table 2). In undifferentiated-type EGCs, a corkscrew pattern was observed in 50 lesions (90.9\%), a fine network pattern in three lesions (5.5\%), and a loop pattern in two lesions (3.6\%). Therefore, the main MV patterns were fine network or loop in differentiated-type EGCs and corkscrew in undifferentiated-type EGCs ( $<<0.001)$.

\section{MS and MV patterns of EGCs according to the invasion depth}

The main MS pattern was oval and/or tubular in mucosal cancers $(67 / 110,60.9 \%)$ and destructive in submucosal cancers $(20 / 50,40.0 \%)(p<0.001)$. There was no difference in the MV patterns between mucosal cancers and submucosal cancers (Fig. 4).

In differentiated-type EGCs, a destructive MS pattern was observed more frequently in submucosal cancers than in mucosal cancers (42.9\% and 10.0\%, respectively, p<0.001) (Table 3, Fig. 5). In undifferentiated-type EGCs, a destructive MS pattern was also observed more frequently in submucosal cancers than in mucosal cancers, but this difference was not statistically significant (33.3\% and 17.5\%, respectively, $\mathrm{p}=0.173$ ). There was no difference in MV patterns between mucosal cancers and submu- cosal cancers in either histologic type.

\section{Association between mucin phenotype and MS and MV patterns}

The most common MS pattern in all four mucin phenotypes was oval and/or tubular. There was no difference in MS patterns according to mucin phenotypes ( $\mathrm{p}=0.287$ ) (Table 4). MV patterns were significantly associated with mucin phenotypes $(\mathrm{p}=0.020)$. The most common MV patterns in G-type and I-type lesions were corkscrew $(41 / 85,48.2 \%)$ and fine network (17/28, 60.7\%), respectively. GI-type lesions exhibited all MV patterns equally.

MS and MV patterns were also analyzed according to the expression of each mucin type (Table 5). Lesions with a papillary or absent MS pattern $(\mathrm{p}=0.021)$ and a corkscrew MV pattern $(p=0.002)$ expressed MUC5AC more frequently than did lesions with other patterns. Lesions with a papillary MS pattern $(p=0.012)$ and a loop MV pattern $(p=0.005)$ expressed MUC6 more frequently than did lesions with other patterns. There was no significant difference in MS patterns according to CD10 expression, but CD10 expression was increased in lesions with a fine network MV pattern compared to lesions with a loop or corkscrew MV pattern $(\mathrm{p}<0.001)$. There was no significant dif- 
Table 3. Magnifying Endoscopy with Narrow Band Imaging of Early Gastric Cancers according to the Invasion Depth of the Tumor

\begin{tabular}{|c|c|c|c|c|c|c|}
\hline & \multicolumn{2}{|c|}{ Differentiated-type } & \multirow[b]{2}{*}{$\mathrm{p}$-value } & \multicolumn{2}{|c|}{ Undifferentiated-type } & \multirow[b]{2}{*}{$\mathrm{p}$-value } \\
\hline & $\begin{array}{c}\text { Mucosal cancer } \\
\quad(\mathrm{n}=70)\end{array}$ & $\begin{array}{c}\text { Submucosal cancer } \\
(n=35)\end{array}$ & & $\begin{array}{c}\text { Mucosal cancer } \\
\quad(\mathrm{n}=40)\end{array}$ & $\begin{array}{c}\text { Submucosal cancer } \\
\qquad(\mathrm{n}=15)\end{array}$ & \\
\hline Microsurface pattern & & & $<0.001$ & & & 0.173 \\
\hline Oval and/or tubular & $52(74.3)$ & $13(37.1)$ & & $15(37.5)$ & $3(20.0)$ & \\
\hline Papillary & $7(10.0)$ & $6(17.1)$ & & 0 & $1(6.7)$ & \\
\hline Destructive & $7(10.0)$ & $15(42.9)$ & & $7(17.5)$ & $5(33.3)$ & \\
\hline Absent & $4(5.7)$ & $1(2.9)$ & & $18(45.0)$ & $6(40.0)$ & \\
\hline Microvascular pattern & & & 0.360 & & & 0.238 \\
\hline Loop & $31(44.3)$ & $12(34.3)$ & & $1(2.5)$ & $1(6.7)$ & \\
\hline Fine network & $33(47.1)$ & $17(48.6)$ & & $1(2.5)$ & $2(13.3)$ & \\
\hline Corkscrew & $6(8.6)$ & $6(17.1)$ & & $38(95.0)$ & $12(80.0)$ & \\
\hline
\end{tabular}

Data are presented as number (\%).
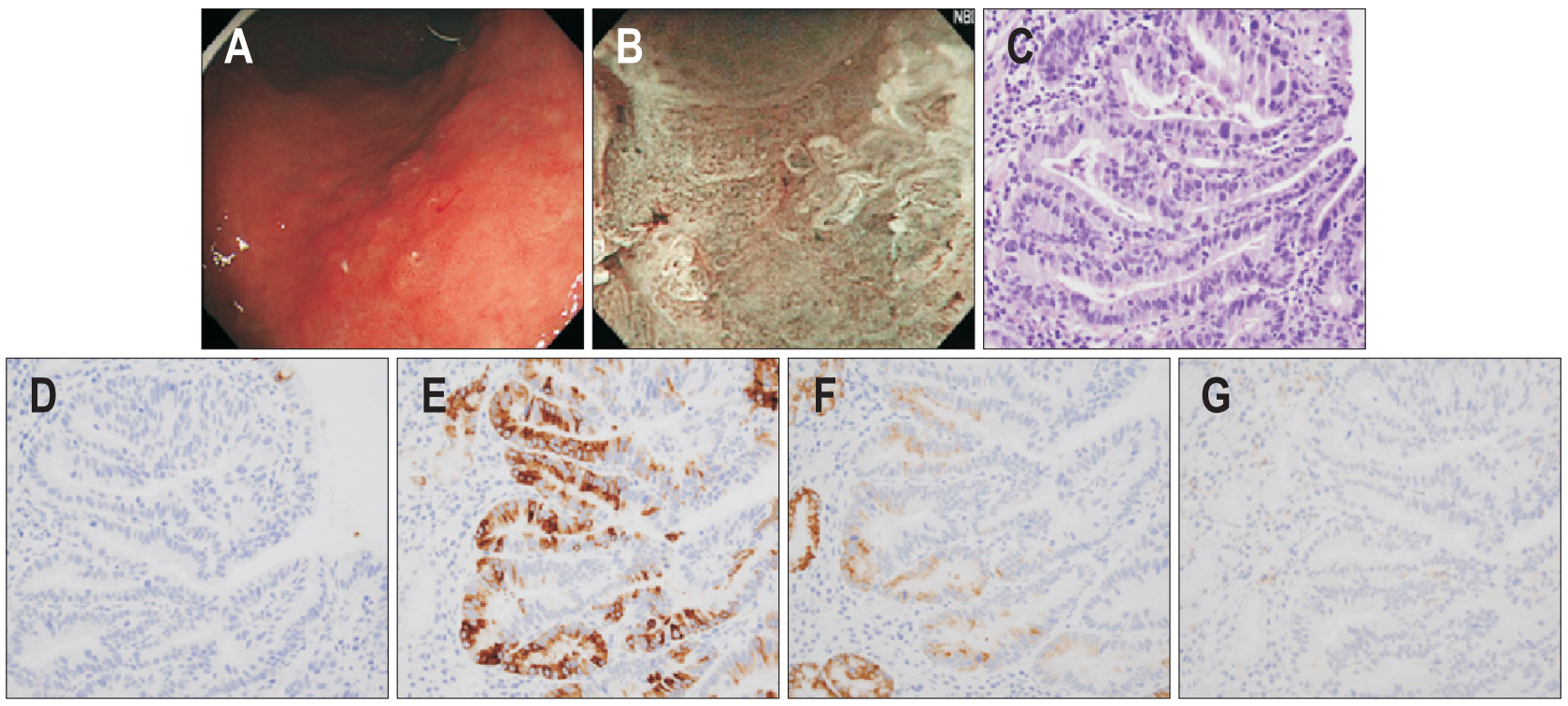

Fig. 5. A case of submucosal cancer. (A) An elevated lesion with a central depression is observed in the mid-body. (B) Magnifying endoscopy with narrow band imaging shows destructive microsurface and fine network microvascular patterns. (C) Histologic examination shows a well-differentiated adenocarcinoma with deep submucosal invasion (H\&E stain, $\times 40$ ). The cancer cells are strongly positive for MUC5AC (E) and MUC6 (F) and negative for MUC2 (D) and CD10 (G).

ference in MS and MV patterns according to MUC2 expression.

\section{DISCUSSION}

In the present study, we classified EGCs on the basis of MS and MV patterns visualized via ME-NBI. We found that the patterns differed according to the histologic type and invasion depth of the tumor. An oval and/or tubular MS pattern and a loop or fine network MV pattern were associated with differentiated-type EGCs, an absent MS pattern and a corkscrew MV pattern with undifferentiated-type EGCs, and a destructive MS pattern with submucosal EGCs.

Several studies have reported the MS or MV patterns in dif- ferent histologic types of EGCs. In an early report examining the MV architecture of differentiated-type adenocarcinoma via $\mathrm{ME}$, the three main findings were disappearance of the regular subepithelial capillary network, a demarcation line, and an irregular MV pattern. ${ }^{1}$ Similar to our findings, a study of MV patterns in depressed-type EGCs demonstrated that fine network and corkscrew patterns were common in differentiated-type and undifferentiated-type carcinomas, respectively. ${ }^{4}$ However, in this study, many lesions had unclassified patterns. To provide classification, a subsequent study focusing on morphological changes in the surface glandular structure of cancerous tissue as well as MV patterns defined two new intralobular loop patterns (ILL-1 and ILL-2). ${ }^{2}$ Although perhaps also present in underlying 
Table 4. Association between Magnifying Endoscopic Findings and Mucin Phenotype in Early Gastric Cancers

\begin{tabular}{|c|c|c|c|c|c|}
\hline & \multicolumn{4}{|c|}{ Mucin phenotype } & \multirow[b]{2}{*}{ p-value } \\
\hline & $\begin{array}{l}\text { Gastric type } \\
\qquad(\mathrm{n}=85)\end{array}$ & $\begin{array}{l}\text { Gastrointestinal type } \\
\qquad(\mathrm{n}=31)\end{array}$ & $\begin{array}{l}\text { Intestinal type } \\
\qquad(\mathrm{n}=28)\end{array}$ & $\begin{array}{l}\text { Null type } \\
(n=16)\end{array}$ & \\
\hline Microsurface pattern & & & & & 0.287 \\
\hline Oval and/or tubular & $44(51.8)$ & $12(38.7)$ & $17(60.7)$ & $10(62.5)$ & \\
\hline Papillary & $9(10.6)$ & $3(9.7)$ & $1(3.6)$ & $1(6.3)$ & \\
\hline Destructive & 13 (15.3) & $9(29.0)$ & 8 (28.6) & $4(25.0)$ & \\
\hline Absent & $19(22.4)$ & $7(22.6)$ & $2(7.1)$ & $1(6.3)$ & \\
\hline Microvascular pattern & & & & & 0.020 \\
\hline Loop & 22 (25.9) & $11(35.5)$ & $5(17.9)$ & $7(43.8)$ & \\
\hline Fine network & 22 (25.9) & $10(32.3)$ & $17(60.7)$ & $4(25.0)$ & \\
\hline Corkscrew & 41 (48.2) & $10(32.3)$ & $6(21.4)$ & 5 (31.3) & \\
\hline
\end{tabular}

Data are presented as number $(\%)$.

Table 5. Association between Magnifying Endoscopic Findings and Mucin Expression in Early Gastric Cancers

\begin{tabular}{|c|c|c|c|c|c|c|c|c|c|}
\hline & \multicolumn{4}{|c|}{ Microsurface pattern } & \multirow[b]{2}{*}{ p-value } & \multicolumn{3}{|c|}{ Microvascular pattern } & \multirow[b]{2}{*}{ p-value } \\
\hline & $\begin{array}{l}\text { Oval and/or } \\
\text { tubular }(\mathrm{n}=83)\end{array}$ & $\begin{array}{l}\text { Papillary } \\
(\mathrm{n}=14)\end{array}$ & $\begin{array}{l}\text { Destructive } \\
(n=34)\end{array}$ & $\begin{array}{l}\text { Absent } \\
(n=29)\end{array}$ & & $\begin{array}{l}\text { Loop } \\
(n=45)\end{array}$ & $\begin{array}{l}\text { Fine network } \\
\qquad(n=53)\end{array}$ & $\begin{array}{c}\text { Corkscrew } \\
(n=62)\end{array}$ & \\
\hline MUC2 expression & & & & & 0.532 & & & & 0.921 \\
\hline Negative & 65 (78.3) & $12(85.7)$ & $24(70.6)$ & $20(69.0)$ & & 33 (73.3) & $41(77.4)$ & $47(75.8)$ & \\
\hline Positive & $18(21.7)$ & $2(14.3)$ & $10(29.4)$ & $9(31.0)$ & & $12(26.7)$ & $12(22.6)$ & $15(24.2)$ & \\
\hline MUC5AC expression & & & & & 0.021 & & & & 0.002 \\
\hline Negative & 31 (37.3) & $2(14.3)$ & $12(35.3)$ & $3(10.3)$ & & $12(26.7)$ & 25 (47.2) & $11(17.7)$ & \\
\hline Positive & $52(62.7)$ & $12(85.7)$ & $22(64.7)$ & 26 (89.7) & & $33(73.3)$ & $28(52.8)$ & $51(82.3)$ & \\
\hline MUC6 expression & & & & & 0.012 & & & & 0.005 \\
\hline Negative & $59(71.1)$ & $6(42.9)$ & 29 (85.3) & $24(82.8)$ & & $27(60.0)$ & $37(69.8)$ & $54(87.1)$ & \\
\hline Positive & $24(28.9)$ & $8(57.1)$ & $5(14.7)$ & $5(17.2)$ & & $18(40.0)$ & $16(30.2)$ & $8(12.9)$ & \\
\hline CD10 expression & & & & & 0.071 & & & & $<0.001$ \\
\hline Negative & 72 (86.7) & $12(85.7)$ & $24(70.6)$ & $27(93.1)$ & & $41(91.1)$ & 36 (67.9) & $58(93.5)$ & \\
\hline Positive & $11(13.3)$ & $2(14.3)$ & $10(29.4)$ & $2(6.9)$ & & 4 (8.9) & $17(32.1)$ & $4(6.5)$ & \\
\hline
\end{tabular}

Data are presented as number $(\%)$.

Negative, $<10 \%$; positive, $\geq 10 \%$.

undifferentiated-type carcinomas, the ILL-1 patterns predicted differentiated-type carcinomas, as did a fine reticular network; a corkscrew pattern predicted undifferentiated-type carcinomas in this study as in our study.

Accurate assessment of submucosal invasion of EGCs is necessary for preoperative indication for ESD. Although the invasion depth of tumor has been evaluated using conventional endoscopy and endoscopic ultrasonography, the diagnostic yields of these approaches are still unsatisfactory. ${ }^{23,24}$ Since it is difficult to increase diagnostic accuracy using only a single modality, findings from different modalities may need to be evaluated in a complementary manner. ME has recently been shown to be effective for determining invasion depth using intrapapillary capillary loop classification of esophageal cancers ${ }^{5,6}$ and pit pat- tern classification of colon cancers. ${ }^{25,26}$ In a study applying the association between irregularly dilated vessels and the invasion depth of colorectal and esophageal cancers to gastric cancers, the presence of dilated vessels ( 3 times larger than irregular microvessels in the lesions) was indicative of submucosal cancer. ${ }^{9}$ However, because this study defined the size of the dilated vessels relative to that of the surrounding irregular microvessels, it was very objective. According to the Kudo's classification of pit patterns determined via magnifying colonoscopy in colorectal cancers, nonstructural pit patterns (i.e., normal tubular pit patterns cannot be identified, termed type $V_{N}$ ) suggest that the cancer involves the submucosal layer. ${ }^{25,26}$ Similarly, a recent study associated nonstructural patterns in gastric cancers with undifferentiated-type histology or submucosal invasion of 
differentiated-type cancers. ${ }^{20}$

In the present study, we divided nonstructural MS patterns into absent and destructive categories based on whether recognizable MS structures were present or not. In tumors with an absent MS pattern, definite MS structures are hardly seen and the area with an absent MS pattern is relatively well-demarcated. In tumors with a destructive MS pattern, MS structures are somewhat seen but are destructed with relatively poorly demarcated margins. We found that the absent MS pattern was mainly observed in undifferentiated-type carcinomas, most notably, signet ring cell carcinomas. The destructive MS pattern was associated with submucosal invasion, and this association was more prominent in differentiated-type than in undifferentiated-type. Therefore, if a destructive MS pattern is observed in EGCs, especially differentiated-type EGCs, endoscopic treatment should be selected very carefully, and the possibility of submucosal invasion should be recognized

The basis for the difference in MS and MV patterns in tumors with different histologic types and with different invasion depths required consideration. Histologically, differentiated-type carcinomas are usually accompanied by a proliferation of vessels within the neoplastic interstitial tissue, whereas undifferentiated-type carcinomas are characterized by the destruction of the normal mucosal vascular architecture by infiltrating cancer cells in the absence of proliferation in the interstitial tissue. ${ }^{27,28}$ These differences presumably contribute to differences in MS and MV patterns; differentiated-type carcinomas display abundant vascular patterns (fine network and loop MV patterns) with maintenance of gland structures (an oval and/or tubular MS pattern), whereas undifferentiated-type carcinomas typically display poor vascular pattern (a corkscrew MV pattern). The absent MS pattern in undifferentiated-type carcinomas could result not only from erosion but also from neoplastic cell infiltration destroying the glandular architecture. ${ }^{29}$ In regard to invasion depth, the deeply invasive portion of differentiated-type carcinomas tends to be less differentiated than the surrounding areas and to exhibit desmoplastic reactions, and the glandular portion of the tumor is less dense. These characteristics may account for the predominance of the destructive MS pattern in submucosal differentiated-type carcinomas.

Despite several studies, ${ }^{12,16,30,31}$ the association of MS and MV patterns with mucin phenotypes has not been fully described. Our study showed that MV (but not MS) patterns varied according to mucin phenotypes. Fine network and corkscrew MV patterns were significantly associated with I-type and Gtype, respectively. When MV patterns were analyzed only in the differentiated-type EGCs, the fine network pattern was predominant in I-type (70.8\%), and the loop and fine network patterns were equally predominant in the G-type $(42.0 \%$ and 40\%, respectively) and GI-type (52.4\% and 47.6\%, respectively) (data not shown). These results are similar to those of a previous study that correlated intralobular loop and fine network patterns with G-type/GI-type and I-type, respectively. ${ }^{19}$ We also found that expression of MUC5AC, MUC6, and CD10 was associated with corkscrew, loop, and fine network MV patterns, respectively. MUC5AC expression was also associated with absent or papillary MS patterns and MUC6 expression with a papillary MS pattern. These findings suggest that expression of specific mucins may account in part for differences in the morphology and vascularization of EGCs and differences in MS and MV patterns visualized via ME-NBI. Further studies are needed to further evaluate the clinical significance of mucin expression patterns predicted by ME-NBI in EGCs.

Our study had some limitations. First, because we performed ME-NBI after gastric cancer was confirmed histologically via endoscopic biopsy, it is possible that MV and especially MS patterns were influenced by biopsy. Endoscopic biopsies often produce prominent mucosal defects with desmoplastic reactions, which could increase the frequency of the destructive MS pattern in mucosal cancers. Second, since gastric cancers are classified as differentiated-type or undifferentiated-type carcinomas on the basis of the predominant histologic type of the tumor, the MS and MV patterns observed in this study may not tell the corresponding histology exactly. Because we included a relatively large number of patients in our study and categorized patterns on the basis of the predominant pattern of the tumor, we could have overcome this limitation at least in part. Third, we could not exactly match each portion of the ME-NBI image to its corresponding portion in the histologic specimens, especially those specimens stained via immunohistochemistry. Prospective studies with one-to-one matching are needed to obtain histological evidence to support our results. Lastly, since the ME-NBI findings were interpreted by a single experienced endoscopist, inter-observer variation was not evaluated. Although ME-NBI findings are thought to be reliable according to recent reports, ${ }^{2,32,33}$ inter-observer variability in the assessment of MENBI findings needs to be evaluated before clinical application.

In conclusion, ME-NBI in EGCs shows distinct differences in MS and MV patterns according to the histologic type and invasion depth of tumor. In addition, MS and MV patterns are associated with mucin phenotypes. Therefore, ME-NBI can be useful for predicting the histopathology and mucin phenotype in EGCs. Further well-designed prospective studies will be needed to verify the clinical significance of ME-NBI findings in EGCs.

\section{CONFLICTS OF INTEREST}

No potential conflict of interest relevant to this article was reported.

\section{ACKNOWLEDGEMENTS}

This study was supported by a grant from the National R\&D Program for Cancer Control, Ministry for Health, Welfare and 
Family Affairs, Korea (0920050) and a grant of the Korea Healthcare technology R\&D Project, Ministry of Health \& Welfare, Republic of Korea (A121994).

\section{REFERENCES}

1. Yao K, Oishi T, Matsui T, Yao T, Iwashita A. Novel magnified endoscopic findings of microvascular architecture in intramucosal gastric cancer. Gastrointest Endosc 2002;56:279-284.

2. Yokoyama A, Inoue H, Minami H, et al. Novel narrow-band imaging magnifying endoscopic classification for early gastric cancer. Dig Liver Dis 2010;42:704-708.

3. Yagi K, Nakamura A, Sekine A, Umezu H. Magnifying endoscopy with narrow band imaging for early differentiated gastric adenocarcinoma. Dig Endosc 2008;20:115-122.

4. Nakayoshi T, Tajiri H, Matsuda K, Kaise M, Ikegami M, Sasaki H. Magnifying endoscopy combined with narrow band imaging system for early gastric cancer: correlation of vascular pattern with histopathology (including video). Endoscopy 2004;36:1080-1084.

5. Yoshida T, Inoue H, Usui S, Satodate H, Fukami N, Kudo SE. Narrow-band imaging system with magnifying endoscopy for superficial esophageal lesions. Gastrointest Endosc 2004;59:288-295.

6. Lee MW, Kim GH, I H, et al. Predicting the invasion depth of esophageal squamous cell carcinoma: comparison of endoscopic ultrasonography and magnifying endoscopy. Scand J Gastroenterol 2014;49:853-861.

7. Saito S, Tajiri H, Ohya T, Nikami T, Aihara H, Ikegami M. Imaging by magnifying endoscopy with NBI implicates the remnant capillary network as an indication for endoscopic resection in early colon cancer. Int J Surg Oncol 2011;2011:242608.

8. Kanao H, Tanaka S, Oka S, Hirata M, Yoshida S, Chayama K. Narrow-band imaging magnification predicts the histology and invasion depth of colorectal tumors. Gastrointest Endosc 2009;69(3 Pt 2):631-636.

9. Kikuchi D, lizuka T, Hoteya S, et al. Usefulness of magnifying endoscopy with narrow-band imaging for determining tumor invasion depth in early gastric cancer. Gastroenterol Res Pract 2013;2013:217695.

10. Kobara H, Mori H, Fujihara S, et al. Prediction of invasion depth for submucosal differentiated gastric cancer by magnifying endoscopy with narrow-band imaging. Oncol Rep 2012;28:841-847.

11. Ha Kim G, Am Song G, Youn Park D, et al. CDX2 expression is increased in gastric cancers with less invasiveness and intestinal mucin phenotype. Scand J Gastroenterol 2006;41:880-886.

12. Kim DH, Shin N, Kim GH, et al. Mucin expression in gastric cancer: reappraisal of its clinicopathologic and prognostic significance. Arch Pathol Lab Med 2013;137:1047-1053.

13. Koseki K, Takizawa T, Koike M, Ito M, Nihei Z, Sugihara K. Distinction of differentiated type early gastric carcinoma with gastric type mucin expression. Cancer 2000;89:724-732.

14. Yamazaki K, Tajima Y, Makino R, et al. Tumor differentiation phenotype in gastric differentiated-type tumors and its relation to tumor invasion and genetic alterations. World J Gastroenterol 2006;12:3803-3809.

15. Toki F, Takahashi A, Aihara R, et al. Relationship between clinicopathological features and mucin phenotypes of advanced gastric adenocarcinoma. World J Gastroenterol 2010;16:2764-2770.

16. Kim GH, Song GA, Park DY, et al. Clinicopathologic significance of gastric and intestinal phenotypic marker expression in gastric carcinomas. Korean J Intern Med 2005;20:191-197.

17. Kocer B, Soran A, Kiyak G, et al. Prognostic significance of mucin expression in gastric carcinoma. Dig Dis Sci 2004;49:954-964.

18. Pinto-de-Sousa J, David L, Reis CA, Gomes R, Silva L, Pimenta A. Mucins MUC1, MUC2, MUC5AC and MUC6 expression in the evaluation of differentiation and clinico-biological behaviour of gastric carcinoma. Virchows Arch 2002;440:304-310.

19. Kobayashi M, Takeuchi M, Ajioka Y, et al. Mucin phenotype and narrow-band imaging with magnifying endoscopy for differentiated-type mucosal gastric cancer. J Gastroenterol 2011;46:10641070.

20. Yoshida T, Kawachi H, Sasajima K, Shiokawa A, Kudo SE. The clinical meaning of a nonstructural pattern in early gastric cancer on magnifying endoscopy. Gastrointest Endosc 2005;62:48-54.

21. Japanese Gastric Cancer Association. Japanese classification of gastric carcinoma: 3rd English edition. Gastric Cancer 2011;14: 101-112.

22. Park do Y, Srivastava A, Kim GH, et al. Adenomatous and foveolar gastric dysplasia: distinct patterns of mucin expression and background intestinal metaplasia. Am J Surg Pathol 2008;32:524533.

23. Choi J, Kim SG, Im JP, Kim JS, Jung HC, Song IS. Endoscopic prediction of tumor invasion depth in early gastric cancer. Gastrointest Endosc 2011;73:917-927.

24. Kwee RM, Kwee TC. The accuracy of endoscopic ultrasonography in differentiating mucosal from deeper gastric cancer. Am J Gastroenterol 2008;103:1801-1809.

25. Kudo S, Tamura S, Nakajima T, Yamano H, Kusaka H, Watanabe H. Diagnosis of colorectal tumorous lesions by magnifying endoscopy. Gastrointest Endosc 1996;44:8-14.

26. Kudo S, Rubio CA, Teixeira CR, Kashida H, Kogure E. Pit pattern in colorectal neoplasia: endoscopic magnifying view. Endoscopy 2001;33:367-373.

27. Ohtani H, Nagura H. Differing microvasculature in the two major types of gastric carcinoma: a conventional, ultrastructural and ultrastructural immunolocalization study of von Willebrand factor. Virchows Arch A Pathol Anat Histopathol 1990;417:29-35.

28. Adachi Y, Mori M, Enjoji M, Sugimachi K. Microvascular architecture of early gastric carcinoma: microvascular-histopathologic correlates. Cancer 1993;72:32-36.

29. Fukui H, Shirakawa K, Nakamura T, et al. Magnifying pharmacoendoscopy: response of microvessels to epinephrine stimulation in differentiated early gastric cancers. Gastrointest Endosc 2006;64:40-44.

30. Tsukashita S, Kushima R, Bamba M, Sugihara H, Hattori T. MUC 
gene expression and histogenesis of adenocarcinoma of the stomach. Int J Cancer 2001;94:166-170.

31. Tajima Y, Yamazaki K, Makino R, et al. Gastric and intestinal phenotypic marker expression in early differentiated-type tumors of the stomach: clinicopathologic significance and genetic background. Clin Cancer Res 2006;12:6469-6479.

32. Anagnostopoulos GK, Yao K, Kaye P, et al. High-resolution mag- nification endoscopy can reliably identify normal gastric mucosa, Helicobacter pylori-associated gastritis, and gastric atrophy. Endoscopy 2007;39:202-207.

33. Pimentel-Nunes P, Dinis-Ribeiro M, Soares JB, et al. A multicenter validation of an endoscopic classification with narrow band imaging for gastric precancerous and cancerous lesions. Endoscopy 2012;44:236-246. 